\title{
LINEAR ELLIPTIC DIFFERENCE INEQUALITIES WITH RANDOM COEFFICIENTS
}

\author{
HUNG-JU KUO AND NEIL S. TRUDINGER
}

\begin{abstract}
We prove various pointwise estimates for solutions of linear elliptic difference inequalities with random coefficients. These estimates include discrete versions of the maximum principle of Aleksandrov and Harnack inequalities and Hölder estimates of Krylov and Safonov for elliptic differential operators with bounded coefficients.
\end{abstract}

\section{INTRODUCTION}

The purpose of this work is to establish analogues for difference operators of certain pointwise estimates for linear elliptic differential operators with bounded, measurable coefficients. In particular we derive discrete versions of the Aleksandrov and Bakel'man maximum principle [1, 2], the Hölder estimates and Harnack inequality of Krylov and Safonov [8], and the local maximum principle and weak Harnack inequalities in Trudinger [14]. Our estimates shall be formulated in such a way that their continuous versions follow via Taylor's formula. In a subsequent paper [9] we apply the Hölder estimate to deduce the convergence of discrete numerical schemes for fully nonlinear elliptic differential equations under very general structure conditions [15]. In fact, the approximation of viscosity solutions of nonlinear elliptic equations provided the motivation for the present study.

The difference operators encompassed are of positive type. To describe them, we let $h$ be a (small) positive parameter and let

$$
\mathbb{Z}_{h}^{n}=\left\{x=\left(m_{1}, \ldots, m_{n}\right) h \mid m_{i} \in \mathbb{Z}, \quad i=1, \ldots, n\right\}
$$

denote the orthogonal lattice or mesh, with mesh length $h$, in Euclidean $n$ space $\mathbb{R}^{n}$. A real-valued function $u$ on $\mathbb{Z}_{h}^{n}$ is called a mesh function, and for fixed $y(\neq 0) \in \mathbb{Z}_{h}^{n}$ we define the following difference operators, acting on the

Received December 28, 1988; revised July 28, 1989.

1980 Mathematics Subject Classification (1985 Revision). Primary 39A10, 39A70, 65N20; Secondary $65 \mathrm{~N} 05,65 \mathrm{~N} 10,35 \mathrm{~J} 15$. 
linear space of mesh functions $\mathscr{M}$ :

$$
\begin{aligned}
& \delta_{y}^{+} u(x)=\frac{1}{|y|}\{u(x+y)-u(x)\}, \\
& \delta_{y}^{-} u(x)=\frac{1}{|y|}\{u(x)-u(x-y)\}, \\
& \delta_{y} u(x)=\frac{1}{2}\left(\delta_{y}^{+}+\delta_{y}^{-}\right) u(x)=\frac{1}{2|y|}\{u(x+y)-u(x-y)\}, \\
& \delta_{y}^{2} u(x)=\delta_{y}^{+} \delta_{y}^{-} u(x)=\frac{1}{|y|^{2}}\{u(x+y)-2 u(x)+u(x-y)\} .
\end{aligned}
$$

We consider second-order difference operators of the form

$$
L_{h} u(x)=\sum_{y} a(x, y) \delta_{y}^{2} u(x)+\sum_{y} b(x, y) \delta_{y} u(x)+c(x) u(x),
$$

with real coefficients $a, b, c$ satisfying (at least)

$$
a(x, y)-\frac{1}{2}|y||b(x, y)| \geq 0, \quad c(x) \leq 0
$$

for all $x, y \in \mathbb{Z}_{h}^{n}$. Such operators are of positive type, as defined by Motzkin and Wasow [11], and satisfy a maximum principle [11, Theorem 3, 4]. Moreover, for any linear, uniformly elliptic, second-order differential operators

$$
L u=a^{i j} D_{i j} u+b^{i} D_{i} u+c u
$$

with bounded coefficients $a^{i j}, b^{i}, c$, satisfying

$$
a^{i j} \xi_{i} \xi_{j} \geq \lambda|\xi|^{2}, \quad c \leq 0
$$

for all $\xi \in \mathbb{R}^{n}$ and some positive constant $\lambda$, there exist difference operators $L_{h}$ satisfying (1.3), which are consistent with $L$ [11, Theorem 2]. We shall supply a proof of this assertion in $\S 6$. The coefficients $a(x, y)$ will also have compact support in $y$ but the reader may even assume for technical simplicity, without losing any basic ideas, that $a(x, y)$ vanishes except when $y=h e_{i}$, $i=1, \ldots, n$, where $e_{1}, \ldots, e_{n}$ are the coordinate vectors. This situation corresponds to a diagonal coefficient matrix $\left[a^{i j}\right]$.

We conclude this introduction with some brief historical remarks. In the special case of two dimensions, a local Hölder (in fact Lipschitz) estimate for solutions of general elliptic difference equations with random coefficients was obtained by Brandt [5]. Elliptic difference operators on graphs, corresponding to divergence form differential operators,

$$
L u=D_{i}\left(a^{i j} D_{j} u\right),
$$

were considered by Merkov [10], who derived Hölder and Harnack inequalities analogous to those of De Giorgi and Moser; see [7, Chapter 8]. The methods in $[5,10]$ are necessarily totally different from ours. Earlier work in the 1960 's dealt with extensions of $\mathbb{L}^{2}$ and Schauder estimates to elliptic difference equations; see for example [3, 4, 13], also [6] for a more modern treatment. Unlike the 
hypotheses of the latter theories, our coefficients are random in the sense that their values at neighboring mesh points are independent of the mesh length.

\section{THE DISCRETE MAXIMUM PRINCIPLE}

In this section, we prove discrete versions of the maximum principles of Aleksandrov [1] and Bakel'man [2]. Our technique adapts the geometric argument of Aleksandrov, as presented for example in [7], to the discrete case. To formulate our result, we let $\Omega$ denote a bounded domain in Euclidean $n$-space $\mathbb{R}^{n}$ and let $\Omega_{h}=\Omega \cap \mathbb{Z}_{h}^{n}$ be the set of mesh points lying in $\Omega$. We shall consider operators of the form (1.2) defined for points $x \in \Omega_{h}$ and satisfying, together with (1.3), a nondegeneracy condition, namely for each point $x \in \Omega_{h}$, there exists an orthogonal set of vectors $y^{1}, \ldots, y^{n} \in \mathbb{Z}_{h}^{n}$ such that

$$
a\left(x, y^{i}\right)-\frac{1}{2}\left|y^{i}\right|\left|b\left(x, y^{i}\right)\right| \geq \lambda_{i}(x)>0 .
$$

Furthermore, we assume the coefficients $a(x, y)$ vanish whenever $|y|=|y|_{2}>$ $N h$ for some $N \in \mathbb{Z}$ and write

$$
\begin{aligned}
& \mathscr{D}=\mathscr{D}(x)=\prod_{i=1}^{n} \lambda_{i}(x), \quad \mathscr{D}^{*}=\mathscr{D}^{1 / n}, \\
& b(x)=\sum_{y}|b(x, y)|, \quad \delta=\operatorname{diam} \Omega+N h .
\end{aligned}
$$

Our maximum principles provide bounds for solutions in terms of integral norms over certain subsets. If $u$ is a mesh function on a set $S \subset \mathbb{Z}_{h}^{n}$, we define the upper contact set of $u$ in $S, \Gamma^{+}=\Gamma_{u}^{+}$, to be the subset of $S$ where $u$ is concave, that is $\Gamma^{+}$consists of those points $x$ for which there exists a hyperplane $P=P(x)$ in $\mathbb{R}^{n+1}$ passing through $x$ and lying above the graph of $u$ in $S$. Denoting $L^{p}$ norms of mesh functions $u$ over sets $S$ by

$$
\|u\|_{p ; S}=\left(\sum_{x \in S} h^{n}|u(x)|^{p}\right)^{1 / p},
$$

we then have the following discrete analogue of [7, Theorem 9.1].

Theorem 2.1. Let $u$ be a mesh function satisfying the difference inequality

$$
L_{h} u \geq f
$$

in $\Omega_{h}$. Then,

$$
\max _{\Omega_{h}} u \leq \max _{\mathbb{Z}_{h}^{n}-\Omega_{h}} u^{+}+C \delta N\left\|f / \mathscr{D}^{*}\right\|_{n, \Gamma^{+}},
$$

where $C$ depends on $n$ and $N\left\|b / \mathscr{D}^{*}\right\|_{n, \Gamma^{+}}, u^{+}=\max \{u, 0\}$, and $\Gamma^{+}$is the upper contact set of $u$ in $\Omega_{h}$.

The proof of Theorem 2.1 is based upon the notion of normal (or supergradient) mapping, the normal mapping of a mesh function $u$ on a set $S$, at a point $x \in S$, being defined by

$$
\chi(x)=\chi_{u}(x)=\left\{p \in \mathbb{R}^{n} \mid u(z) \leq u(x)+p \cdot(z-x) \text { for all } z \in S\right\} .
$$


The upper contact set $\Gamma^{+}$of $u$ on $S$ is thus the subset of $S$ where $\chi$ is nonempty. If $S^{\prime}$ is any subset of $S$, we further define

$$
\chi\left(S^{\prime}\right)=\bigcup_{x \in S^{\prime}} \chi(x)
$$

Defining the boundary $\partial S$ of a subset $S \subset \mathbb{Z}_{h}^{n}$ to be the set of points in $S$ which have a neighbor outside $S$ and the interior $\stackrel{\circ}{S}=S-\partial S$, we then have as a preliminary estimate:

Lemma 2.2. Let $u$ be a mesh function on $S \subset \mathbb{Z}_{h}^{n}$ satisfying $u=0$ on $\partial S$, $\max _{S} u>0$. Then,

$$
B_{R}=\left\{p \in \mathbb{R}^{n}|| p \mid<R\right\} \subset \chi_{u}(\stackrel{\circ}{S})
$$

for $R=\max _{S} u / \operatorname{diam} S$.

Proof. The proof of (2.7) corresponds to that in the nondiscrete case as given, for example, in [7, p. 222]. Suppose that $u$ takes a positive maximum at a point $y \in \stackrel{\circ}{S}$, and let $u_{y}$ be the mesh function on $\partial S \cup\{y\}$ given by

$$
u_{y}(x)= \begin{cases}u(y) & \text { if } x=y, \\ 0 & \text { if } x \in \partial S\end{cases}
$$

Then $\chi_{u_{v}}(y) \subset \chi_{y}(\stackrel{\circ}{S})$, since for each hyperplane passing through $(y, u(y))$ and lying above $\partial S \times\{0\}$, there will exist a parallel hyperplane passing through some point in the graph of $u$ on $\stackrel{\circ}{S}$ and lying above $u$ on $S$. Letting $k$ be the function on $\mathbb{R}^{n}$ whose graph is the cone $K$ with vertex $(y, u(y))$ and base $B_{d}(y)$, where $d=\operatorname{diam} \Omega$, we then have (as in [7, p. 222])

$$
B_{R}=\chi_{k}(y) \subset \chi_{u_{y}}(y) \subset \chi_{u}(\stackrel{\circ}{S}) \text {. }
$$

Proof of Theorem 2.1. We first treat the case $b \equiv 0$. Setting

$$
S=\left\{x+y\left|x \in \Omega_{h},\right| y \mid \leq N h\right\},
$$

we can assume, by replacing $u$ with $u-\max _{S-\Omega_{h}} u^{+}$, that $u \leq 0$ on $\partial S$ and $\max _{S} u>0$ (otherwise (2.5) is trivial). Writing $v=u^{+}$, we then have $\Gamma_{v}^{+} \subset \Omega_{h} \cap \Gamma_{u}^{+}$, with $u=v$ on $\Gamma^{+}$. Consequently, for $x \in \Gamma_{v}^{+}$and $|y| \leq N h$, we have

for some $p \in \mathbb{R}^{n}$, whence by addition

$$
u(x \pm y) \leq u(x) \pm p \cdot y
$$

$$
\delta_{y}^{2} u(x) \leq 0
$$

Hence, for (2.4) and (1.3),

$$
\begin{aligned}
0 & \leq a(x, y)[2 v(x)-v(x+y)-v(x-y)] \\
& \leq a(x, y)[2 u(x)-u(x+y)-u(x-y)] \\
& \leq|y|^{2} \sum_{y^{\prime}} a\left(x, y^{\prime}\right) \delta_{y^{\prime}}^{2} u(x) \leq|y|^{2}(c u(x)-f(x)) \leq|y|^{2}|f(x)| .
\end{aligned}
$$


In particular, for $y=y^{i}$, we have

$$
\left[v(x)-v\left(x-y^{i}\right)\right]-\left[v\left(x+y^{i}\right)-v(x)\right] \leq\left|y^{i}\right|^{2}|f(x)| / \lambda_{i},
$$

so that, if $p \in \chi_{v}(x)$, then $p_{y^{i}}=p \cdot y^{i} /\left|y^{i}\right|$ lies between the numbers $\delta_{y^{i}}^{+} v(x)$ and $\delta_{y^{i}}^{+} v(x)+\left|y^{i}\right||f(x)| / \lambda_{i}$. Accordingly, the $n$-dimensional Lebesgue measure of $\chi_{v}(x)$ is estimated by

$$
\left|\chi_{v}(x)\right| \leq \frac{\prod\left|y^{i}\right|}{\mathscr{D}(x)}|f(x)|^{n} \leq \frac{(N h|f(x)|)^{n}}{\mathscr{D}(x)} .
$$

Thus, by Lemma 2.2 , with $\omega_{n}$ denoting the volume of the unit ball in $\mathbb{R}^{n}$,

$$
\omega_{n}\left(\frac{1}{\delta} \max _{S} v\right)^{n} \leq\left|\chi_{v}\left(\Omega_{h}\right)\right| \leq \sum_{x \in \Gamma^{+}} \frac{(N h|f(x)|)^{n}}{\mathscr{D}(x)},
$$

whence we obtain the special case of $(2.5)$,

$$
\max _{\Omega_{h}} u \leq \max _{S-\Omega_{h}} u^{+}+\frac{\delta N}{\omega_{n}^{1 / n}}\left\|\frac{f}{\mathscr{D}^{*}}\right\|_{n, \Gamma^{+}} .
$$

To treat the general case, we introduce the function

$$
g(\mathbf{p})=\left(|\mathbf{p}|^{n /(n-1)}+\mu^{n /(n-1)}\right)^{1-n}
$$

for $\mathbf{p} \in \mathbb{R}^{n}$ and some $\mu>0$ to be fixed later. By Lemma 2.2, we have for $R=\frac{1}{\delta} \max _{S} v$ the estimate

$$
\int_{B_{R}} g \leq \int_{\chi_{v}\left(\Omega_{h}\right)} g \leq \sum_{x \in \Gamma_{v}^{+}}\left\{\max _{\chi_{v}(x)} g\right\}\left|\chi_{v}(x)\right| \leq \sum_{x \in \Gamma_{v}^{+}} g(\mathbf{p})\left|\chi_{v}(x)\right|,
$$

where $\mathbf{p}=\mathbf{p}(x) \in \chi_{v}(x)$ is chosen so that $g(\mathbf{p})=\max _{\chi_{v}(x)} g$, which is possible since $\chi_{v}(x)$ is a closed set. Now, since $\mathbf{p}_{y}=\mathbf{p} \cdot y /|y|$ lies between $\delta_{y}^{+} u(x)$ and $\delta_{y}^{-} u(x)$, there exists a number $r=r_{\mathbf{p}} \in[0,1]$ such that

$$
\mathbf{p}_{y}=(1-r) \delta_{y}^{+} u(x)+r \delta_{y}^{-} u(x) \text {. }
$$

Consequently, using the differential inequality (2.4), we have (for $x \in \Gamma_{v}^{+}$)

$$
\begin{aligned}
-\sum_{y} a(x, y) \delta_{y}^{2} u(x) & \leq \sum_{y} b(x, y) \delta_{y} u(x)+c(x) u(x)-f(x) \\
& \leq \frac{1}{2} \sum_{y} b(x, y)\left(\delta_{y}^{+} u(x)+\delta_{y}^{-} u(x)\right)-f(x) \\
& =\sum_{y} b(x, y)\left\{\mathbf{p}_{y}+\left(r-\frac{1}{2}\right)|y| \delta_{y}^{2} u(x)\right\}-f(x) .
\end{aligned}
$$

Hence, defining new coefficients

$$
\bar{a}(x, y)=a(x, y)-\frac{1}{2}|y||b(x, y)|,
$$


we therefore obtain in place of (2.9),

$$
\begin{aligned}
\bar{a}(x, y)[2 v(x)-v(x+y)-v(x-y)] \\
\leq|y|^{2}(b(x)|\mathbf{p}|+|f(x)|) \leq|y|^{2} \frac{\left(|b|^{n}+\mu^{-n}|f|^{n}\right)^{1 / n}}{g^{1 / n}(\mathbf{p})}
\end{aligned}
$$

by Hölder's inequality. We thus obtain, in place of $(2.11)$, the estimate

$$
\left|\chi_{v}(x)\right| \leq \frac{(N h)^{n}\left(|b|^{n}+\mu^{-n}|f|^{n}\right)}{g(\mathbf{p}) \mathscr{D}} .
$$

Thus, by (2.14),

$$
\begin{aligned}
\int_{B_{R}} g & \leq \sum_{x \in \Gamma_{v}^{+}} g(\mathbf{p})\left|\chi_{v}(x)\right| \leq(N h)^{n} \sum_{x \in \Gamma_{v}^{+}}\left(|b|^{n}+\mu^{-n}|f|^{n}\right) / \mathscr{D} \\
& \leq\left\{N\left\|b / \mathscr{D}^{*}\right\|_{n, \Gamma^{+}}\right\}^{n}+1 \equiv \bar{b}
\end{aligned}
$$

with the choice $\mu=N\left\|f / \mathscr{D}^{*}\right\|_{n, \Gamma^{+}}$for $f \not \equiv 0$.

Using the proof of [6, Theorem 9.1], we then obtain the estimate

$$
\max v \leq\left\{\exp \left(\frac{2^{n-2} \bar{b}}{\omega_{n}}\right)-1\right\}^{1 / n} \delta N\left\|f / \mathscr{D}^{*}\right\|_{n, \Gamma^{+}},
$$

and the proof of Theorem 2.1 is thus complete.

Remarks. (i) We need only assume the vectors $y^{1}, \ldots, y^{n}$ are linearly independent in condition (2.1), in which case we replace $\mathscr{D}$ in $(2.2)$ by

$$
\mathscr{D}=\left(\prod_{i=1}^{n} \lambda_{i}(x)\right)\left|\operatorname{det}\left[y_{j}^{i} /\left|y^{i}\right|\right]\right| \text {. }
$$

(ii) The constants in estimates (2.12) and (2.18) may be improved. In particular, using in place of $(2.10)$ the sum

$$
\sum_{i=1}^{n} \lambda_{i}\left\{\delta_{y^{i}}^{-} v(x)-\delta_{y^{i}}^{+} v(x)\right\} \leq N h|f(x)|,
$$

we obtain, instead of $(2.11)$,

$$
\left|\chi_{v}(x)\right| \leq \frac{(N h|f(x)|)^{n}}{n^{n} \mathscr{D}(x)},
$$

so that $\mathscr{D}^{*}$ can be replaced by $n \mathscr{D}^{*}$ in $(2.12)$ and (2.18).

\section{THE DISCRETE LOCAL MAXIMUM PRINCIPLE}

In this section, we prove a discrete version of the local maximum principle (or mean value inequality) in [14, Theorem 1]. Although our method is inspired by that in [14], the manipulation of the cutoff functions in the discrete case is 
far more intricate. We shall maintain the same operator hypotheses as in the preceding section but now write, instead of (2.2),

$$
\begin{aligned}
& \lambda(x)=\inf \lambda_{i}(x), \quad a(x)=\sum_{y} a(x, y), \\
& a_{0}=\sup a / \lambda, \quad b_{0}=\sup b / \lambda, \quad c_{0}=\sup |c| / \lambda .
\end{aligned}
$$

Theorem 3.1. Let $u$ be a mesh function satisfying the difference inequality (2.4) in $\Omega_{h}$. Then for any ball $B=B_{R}\left(x_{0}\right) \subset \Omega$ with center $x_{0} \in \Omega_{h}, N h \leq R$, concentric subball $B^{\sigma}=B_{\sigma R}\left(x_{0}\right), 0<\sigma<1$, and exponent $p>0$, we have the estimate

$$
\max _{B_{h}^{\sigma}} u \leq C\left\{\left[\left(\frac{h}{R}\right)^{n} \sum_{B_{h}}\left(u^{+}\right)^{p}\right]^{1 / p}+R\left\|\frac{f}{\lambda}\right\|_{n ; B_{h}}\right\},
$$

where $B_{h}=B \cap \Omega_{h}, B_{h}^{\sigma}=B^{\sigma} \cap B_{h}$, and the constant $C$ depends on $n, N, \sigma$, $p, a_{0}$, and $b_{0} R$.

Proof. Without loss of generality, we can assume $x_{0}=0$. Similarly to [14], we shall consider a function of the form

$$
v=\eta \mathcal{u},
$$

where $\eta$ is an appropriate cutoff function for the ball $B$ with $\eta>0$ in $B$ and $\eta=0$ otherwise. First, we observe that if $x$ is a point in the upper contact set of $v$ in $B_{h}$, then there exists a vector $\mathbf{p} \in \chi_{v}(x)$ such that

$$
|\mathbf{p}| \leq v(x) /\left(R-|x|_{\infty}\right) \text {. }
$$

Furthermore, for any $y \in \mathbb{Z}_{h}^{n}-\{0\}$ (with $a(x, y) \neq 0$ ), we can write

$$
\mathbf{p}_{y}=\frac{\mathbf{p} \cdot y}{|y|}=r_{y} \delta_{y}^{+} v(x)+\left(1-r_{y}\right) \delta_{y}^{-} v(x)
$$

for some $r_{y}=r_{y}(x) \in[0,1]$. By rewriting

we then obtain

$$
\mathbf{p}_{y}=-r_{y}\left(\delta_{y}^{-}-\delta_{y}^{+}\right) v+\delta_{y}^{-} v=\left(1-r_{y}\right)\left(\delta_{y}^{-}-\delta_{y}^{+}\right) v+\delta_{y}^{+} v,
$$

$$
\begin{aligned}
&\left\{r_{y} \eta(x+y)+\left(1-r_{y}\right) \eta(x-y)\right\}\left(\delta_{y}^{-}-\delta_{y}^{+}\right) v(x) \\
&= {[\eta(x+y)-\eta(x-y)] \mathbf{p}_{y}+\eta(x-y) \delta_{y}^{-} v(x)-\eta(x+y) \delta_{y}^{+} v(x) } \\
&=-2|y| \delta_{y} \eta(x) \mathbf{p}_{y}+\eta(x) u(x)[\eta(x+y)+\eta(x-y)] \\
&-\eta(x+y) \eta(x-y)[u(x+y)+u(x-y)] \\
&=-2|y| \delta_{y} \eta(x) \mathbf{p}_{y}-\eta(x+y) \eta(x-y)[u(x+y)+u(x-y)-2 u(x)] \\
&+u(x)\{\eta(x)[\eta(x+y)+\eta(x-y)]-2 \eta(x+y) \eta(x-y)\} .
\end{aligned}
$$

Consequently, we have the formula

$$
\begin{aligned}
-\left\{r_{y} \eta(x+y)+\left(1-r_{y}\right) \eta(x-y)\right\} \delta_{y}^{2} v(x) \\
=2 \mathbf{p}_{y} \delta_{y} \eta(x)-\eta(x+y) \eta(x-y) \delta_{y}^{2} u(x) \\
+\left[2 \delta_{y}^{+} \eta(x) \delta_{y}^{-} \eta(x)-\eta(x) \delta_{y}^{2} \eta(x)\right] u(x) .
\end{aligned}
$$


By a similar argument, we also have

$$
\begin{aligned}
-|y| & \left\{r_{y} \eta(x+y)-\left(1-r_{y}\right) \eta(x-y)\right\} \delta_{y}^{2} v(x) \\
= & {[\eta(x+y)+\eta(x-y)] \mathbf{p}_{y}+2 \eta(x+y) \eta(x-y) \delta_{y} u(x) } \\
& +2 \eta(x) u(x) \delta_{y} \eta(x) .
\end{aligned}
$$

To proceed further, we assume $\eta(x+y) \eta(x-y)>0$, so that, combining (3.7) and (3.8) with (2.4) and writing, as before,

$$
\bar{a}(x, y)=a(x, y)-\frac{1}{2}|y||b(x, y)|,
$$

we obtain the following difference inequality for $v$ :

$$
\begin{aligned}
-\sum & \bar{a}(x, y)\left\{\frac{r_{y}}{\eta(x+y)}+\frac{\left(1-r_{y}\right)}{\eta(x-y)}\right\} \delta_{y}^{2} v(x) \\
\leq & \sum a(x, y)\left\{\frac{2 \mathbf{p}_{y} \delta_{y} \eta(x)+\left[2 \delta_{y}^{+} \eta(x) \delta_{y}^{-} \eta(x)-\eta(x) \delta_{y}^{2} \eta(x)\right] u(x)}{\eta(x+y) \eta(x-y)}\right\} \\
& -\sum b(x, y)\left\{\left[\frac{1}{\eta(x+y)}+\frac{1}{\eta(x-y)}\right] \frac{\mathbf{p} y_{y}}{2}+\frac{\eta(x) u(x) \delta_{y} \eta(x)}{\eta(x+y) \eta(x-y)}\right\} \\
& -f(x) .
\end{aligned}
$$

We now fix $\eta$ by setting

$$
\eta(x)=\left(1-\frac{|x|^{2}}{R^{2}}\right)^{\beta}, \quad x \in B,
$$

for some $\beta \geq 2$ to be chosen later. Then, provided

$$
R^{2}-|x|^{2} \geq 4 R N h
$$

we have the inequalities

$$
\begin{aligned}
2^{-\beta} & \leq \frac{\eta(x \pm y)}{\eta(x)} \leq 2^{\beta}, \\
\left|\delta_{y}^{ \pm} \eta\right| & \leq \frac{\beta 2^{\beta}}{R} \eta^{1-1 / \beta}(x), \\
\left|\delta_{y}^{2} \eta\right| & \leq \frac{\beta(\beta-1) 2^{\beta}}{R^{2}} \eta^{1-2 / \beta}(x),
\end{aligned}
$$

so that, using these inequalities together with (3.4) in (3.9), we obtain

$$
\begin{aligned}
-\bar{L} v(x) & \equiv-\sum \bar{a}(x, y) \delta_{y}^{2} v(x) \\
& \leq C\left\{a(x) \frac{\eta^{1-2 / \beta}}{R^{2}} u(x)+b(x) \frac{\eta^{1-1 / \beta}}{R} u(x)+\eta|f(x)|\right\},
\end{aligned}
$$


where $C$ depends only on $\beta$ and $N$. If the restriction (3.11) on the point $x$ is not satisfied, we first observe that, by replacing $u$ by $u^{+}$, we can assume $u \geq 0$, whence

$$
-\delta_{y}^{2} v(x) \leq \frac{2 \eta(x) u(x)}{h^{2}} \leq \frac{32 N^{2}}{R^{2}} u(x) \eta^{1-2 / \beta}(x),
$$

using $\eta^{2 / \beta} \leq(4 N h / R)^{2}$, so that (3.12) continues to hold. The remainder of the proof now follows along the lines of [14]. Applying the discrete maximum principle, Theorem 2.1, to the function $v$, we obtain

$$
\max _{B_{h}} v \leq C\left\{\frac{a_{0}+b_{0} R}{R}\left\|\eta^{1-2 / \beta} u^{+}\right\|_{n ; B_{h}}+R\left\|\frac{f}{\lambda}\right\|_{n ; B_{h}}\right\},
$$

where $C$ depends on $n, N$, and $\beta$; with the choice $\beta=2 n / p$ (assuming $p \leq n)$ we finally arrive at the estimate (3.2).

Remarks. (i) There is no need to require $c \leq 0$ in (1.3); the constant $C$ in (3.2) then depends also on $c_{0} R^{2}$.

(ii) When $f \equiv 0, p=1$, and $u \geq 0$ in (3.2), we obtain the mean value inequality

$$
u\left(x_{0}\right) \leq \frac{C}{\left[B_{h}\right]} \sum_{B_{h}} u(x),
$$

where $C$ depends on $n, N, a_{0}$, and $b_{0} R$, and $\left[B_{h}\right]$ denotes the number of mesh points in $B_{h}$. The estimate (3.14) is well known in the special case $y^{i}=h e_{i}, a\left(x, y^{i}\right)=1, a(x, y)=0$ for $y \neq y^{i}, i=1, \ldots, n, b(x, y)=0$, $c=0$, when $L_{h}$ is the discrete Laplacian.

\section{HARNACK AND HöLDER ESTIMATES}

In this section, we proceed from the local maximum principle, Theorem 3.1, to derive interior Harnack and Hölder estimates for solutions of elliptic difference equations. Our approach is modelled on that suggested in [7, Problem 9.11], with a discrete version of the fundamental Krylov-Safonov covering argument playing a key role. First we require some lower estimates for positive supersolutions, and for these we continue the same hypotheses and notation as in the preceding section.

Lemma 4.1. Let $u$ be a mesh function satisfying the difference inequality

$$
L_{h} u \leq f
$$

in $\Omega_{h}$, with $u \geq 0$ on $B_{h}$, where $B=B_{R}\left(x_{0}\right) \subset \Omega, x_{0} \in \Omega_{h}$. Then, for any concentric subballs $B^{\sigma}=B_{\sigma R}\left(x_{0}\right), B^{\tau}=B_{\tau R}\left(x_{0}\right), \sigma \leq \tau<1$, and sufficiently small $h$, we have the estimate

$$
\min _{B_{h}^{r}} u \geq \gamma \min _{B_{h}^{n}} u-C R\left\|\frac{f}{\lambda}\right\|_{n: B_{h}},
$$


where $\gamma$ and $C$ are positive constants depending on $n, \sigma, \tau, N, a_{0}, b_{0} R$, and $c_{0} R^{2}$.

Proof. Again, we may assume $x_{0}=0$. Setting $\eta(x)=\left(R^{2}-|x|^{2}\right)^{\beta}$ for $|x| \leq R$, $\beta \geq 2$, and $\eta=0$ for $|x| \geq R$, we have by differentiation,

$$
\begin{aligned}
D \eta & =-2 \beta x\left(R^{2}-|x|^{2}\right)^{\beta-1} \\
D_{i j} \eta(x) & =-2 \beta \delta_{i j}\left(R^{2}-|x|^{2}\right)^{\beta-1}+4 \beta(\beta-1) x_{i} x_{j}\left(R^{2}-|x|^{2}\right)^{\beta-2}
\end{aligned}
$$

for $|x| \leq R$, so that

$$
\begin{aligned}
L \eta(x) \equiv & \sum_{y}\left\{\frac{a(x, y)}{|y|^{2}} \sum_{i, j} y_{i} y_{j} D_{i j} \eta+\frac{b(x, y)}{|y|} \sum_{i} y_{i} D_{i} \eta\right\}+c \eta \\
\geq & \left\{4 \beta(\beta-1) \lambda|x|^{2}-2 \beta(a+b|x|)\left(R^{2}-|x|^{2}\right)+c R^{2}\left(R^{2}-|x|^{2}\right)\right\} \\
& \cdot\left(R^{2}-|x|^{2}\right)^{\beta-2} \\
\geq & 0
\end{aligned}
$$

for $\sigma R \leq|x| \leq R$ and $\beta \geq\left(a_{0}+b_{0} R+c_{0} R^{2}\right) / \sigma^{2} \quad\left(\equiv \beta_{0}\right)$. Now, writing $m=\min _{B_{h}^{\sigma}} u$ and

$$
w=m R^{-2 \beta} \eta-u,
$$

we have $w(x) \leq 0$ for $|x|<\sigma R,|x|>R$, and

$$
L_{h} w \geq m R^{-2 \beta}\left(L_{h} \eta-L \eta\right)-f
$$

in $B_{h}-B_{h}^{\sigma}$. Consequently, by the maximum principle, Theorem 2.1 , we obtain

$$
w \leq C_{0} \frac{m h}{R}+C(R+N h) N\left\|\frac{f}{\lambda}\right\|_{n ; B_{h}},
$$

where $C_{0}$ is a constant depending only on $n, \beta, a_{0}, b_{0} R$, and $N$, and $C$ depends on $n$ and $b_{0} R$. Hence it follows that in $B_{h}^{\tau}$,

$$
u \geq\left[\left(1-\tau^{2}\right)^{\beta}-\frac{C_{0} h}{R}\right] m-C(R+N h) N\left\|\frac{f}{\lambda}\right\|_{n ; B_{h}},
$$

which implies (4.2) for

$$
\frac{C_{0} h}{R} \leq \gamma=\frac{1}{2}\left(1-\tau^{2}\right)^{\beta}
$$

Using Theorem 3.1, we now improve Lemma 4.1. For convenience we shall take $c=0$, but the general case may be recovered in the final Hölder estimates by replacing $f$ by $f-c u$.

Lemma 4.2. Under the hypotheses of Lemma 4.1, there exists a constant $\delta<1$, depending on $n, N, a_{0}$, and $b_{0} R$, such that, if $\Gamma \subset B_{h}^{\sigma}$ satisfies

$$
[\Gamma] \geq \delta\left[B_{h}^{\sigma}\right],
$$


then

$$
\min _{B_{n}^{\tau}} u \geq \gamma \min _{\Gamma} u-C R\left\|\frac{f}{\lambda}\right\|_{n ; B_{h}},
$$

where $\gamma, h$, and $C$ are controlled by the same quantities as in (4.2).

Proof. Setting $m=\min _{\Gamma} u$, we apply Theorem 3.1 to the function $v=m-u$ in the balls $B^{\sigma / 2}, B^{\sigma}$ to obtain

$$
\begin{aligned}
\max _{B_{h}^{\sigma / 2}} v & \leq C\left\{\frac{1}{\left[B_{h}^{\sigma}\right]} \sum_{B_{h}^{\sigma}} v^{+}+R\left\|\frac{f}{\lambda}\right\|_{n ; B_{h}}\right\} \\
& \leq C\left\{(1-\delta) \max _{B_{h}^{\sigma}} v+R\left\|\frac{f}{\lambda}\right\|_{n ; B_{h}}\right\},
\end{aligned}
$$

where $C$ depends on $n, N, a_{0}, b_{0} R$. Consequently,

$$
m-\min _{B_{h}^{\sigma / 2}} u \leq C\left\{(1-\delta)\left(m-\min _{B_{h}^{\sigma}} u\right)+R\left\|\frac{f}{\lambda}\right\|_{n ; B_{h}}\right\},
$$

so that for

$$
\delta=1-1 / 2 C
$$

we have

$$
\min _{B_{h}^{\sigma / 2}} u \geq \frac{m}{2}-C R\left\|\frac{f}{\lambda}\right\|_{n ; B_{h}},
$$

whence (4.10) follows from Lemma 4.1.

We can now proceed to the derivation of a weak Harnack inequality for nonnegative supersolutions, which constitutes yet a further improvement of Lemma 4.1. In order to remove the restriction (4.8) on the mesh length $h$ in Lemmas 4.1 and 4.2, we need to assume that mesh points are effectively related by the operator $L_{h}$, and this is readily accomplished by requiring, as we do henceforth, that the vectors $y^{i}$ in condition $(2.1)$ be given by $y^{i}=h e_{i}$, where $e_{i}$, $i=1, \ldots, n$, are the coordinate vectors.

Theorem 4.3. Let $u$ be a mesh function satisfying the difference inequality (4.1) in $\Omega_{h}$, with $u \geq 0$ in $B_{h}$, where $B=B_{R}\left(x_{0}\right) \subset \Omega, x_{0} \in \Omega_{h}$. Then there exists a constant $p>0$, depending on $n, N, a_{0}, b_{0} R$, such that, for any $\tau<1$, $N h<(1-\tau) R$, we have

$$
\left\{\left(\frac{h}{R}\right)^{n} \sum_{B_{h}^{\tau}} u^{p}\right\}^{1 / p} \leq C\left\{\min _{B_{h}^{\tau}} u+R\left\|\frac{f}{\lambda}\right\|_{n ; B_{h}}\right\},
$$

where $C$ depends on $n, N, a_{0}, b_{0} R, \tau$.

Proof. Let us first observe that the restriction (4.8) can be replaced by the condition $N h<(1-\tau) R$. To see this, we fix any point $z$ in $B_{h}^{\tau}$, so that $u(x) \geq 0$ for 
$|x-z|<N h$, whence by virtue of the difference inequality (4.1) and condition (2.1) with $y^{i}=h e_{i}$,

$$
2 a(z) u(z) \geq \lambda_{i} u\left(z \pm h e_{i}\right)-h^{2} f(x), \quad i=1, \ldots, n .
$$

Consequently, if $R \leq C_{0} h / \gamma$, the result of Lemma 4.1 follows by a finite iteration (depending only on $n, C_{0}, \tau$ ).

To complete the proof of Theorem 4.3, we follow an argument analogous to the original measure-theoretic argument of Krylov and Safonov [8]. We shall denote the cube of side length $2 R$ and center $x_{0} \in \mathbb{R}^{n}$, parallel to the coordinate axes, by $Q_{R}\left(x_{0}\right)$, that is

$$
Q_{R}\left(x_{0}\right)=\left\{x \in \mathbb{R}^{n}|| x-\left.x_{0}\right|_{\infty} \leq R\right\} .
$$

Let us now fix a cube $Q=Q_{2^{k} h}\left(x_{0}\right)$ satisfying

$$
\left\{\begin{array}{l}
x_{0}-(h / 2, h / 2, \ldots, h / 2) \in \mathbb{Z}_{h}^{n}, \quad k \in \mathbb{N}, \\
N h \leq 2^{k} h \leq \operatorname{dist}\left(x_{0}, \partial \Omega\right) / 3 \sqrt{n} .
\end{array}\right.
$$

Defining $\Gamma_{t}=\left\{x \in Q_{h} \mid u(x)>t\right\}$ for $t>0$, we then obtain from Lemma 4.2, with judicious choice of $\sigma, \tau$, that for any parallel subcube $Q^{\prime}=Q_{r}(z)$, $z \in \mathbb{Z}_{h / 2}^{n}$, satisfying

$$
\left[\Gamma_{t} \cap Q^{\prime}\right] \geq \delta\left[Q_{h}^{\prime}\right]
$$

we have

$$
u(x) \geq \gamma t-C R\left\|\frac{f}{\lambda}\right\|_{n ; B_{h}}
$$

in $Q_{h} \cap Q_{3 r}(z)$, where $\gamma$ and $C$ are positive constants depending on $n, N, a_{0}$, $b_{0} R$. We next invoke the following discrete covering lemma, whose proof is a replica of the continuous case [14, Lemma 3]. Note that our choice of $x_{0}$ is significant here.

Lemma 4.4. For $\Gamma \subset Q_{h}$ and $0<\delta<1$, set

$$
\Gamma_{\delta}=\bigcup_{Q^{\prime}}\left\{Q_{3 r}(z) \cap Q_{h} \mid\left[\Gamma \cap Q^{\prime}\right] \geq \delta\left[Q_{h}^{\prime}\right]\right\},
$$

where $Q^{\prime}=Q_{r}(z), \quad z \in \mathbb{Z}_{h / 2}^{n}$. Then either $\Gamma_{\delta}=Q_{h}$ or $\left[\Gamma_{\delta}\right] \geq \delta^{-1}[\Gamma]$.

From Lemma 4.4, it follows by induction that, if $\left[\Gamma_{t}\right] \geq \delta^{s}\left[Q_{h}\right], s \in \mathbb{N}$, then

$$
u(x) \geq \gamma^{s} t-C R\left\|\frac{f}{\lambda}\right\|_{n ; B_{h}}
$$

for all $x \in Q_{h}$. Choosing $s$ so that $\delta^{s} \leq\left[\Gamma_{t}\right] /\left[Q_{h}\right] \leq \delta^{s-1}$, that is

$$
s \geq \frac{1}{\log \delta} \log \left(\frac{\left[\Gamma_{t}\right]}{\left[Q_{h}\right]}\right) \geq s-1,
$$


we then have

$$
\inf _{Q_{h}} u \geq \gamma_{t}\left(\frac{\left[\Gamma_{t}\right]}{\left[Q_{h}\right]}\right)^{\log \gamma / \log \delta} .
$$

For fixed $p<\log \delta / \log \gamma$, we thus obtain

$$
\left\{\frac{1}{\left[Q_{h}\right]} \sum_{Q_{h}} u^{p}\right\}^{1 / p} \leq C\left\{\min _{Q_{h}} u+R\left\|\frac{f}{\lambda}\right\|_{n ; B_{h}}\right\},
$$

where $C$ depends on $n, N, a_{0}$, and $b_{0} R$. The weak Harnack inequality, (4.13), now follows directly from (4.19), provided $\tau$ is sufficiently small. But then the general case may be deduced by a standard chaining argument.

By combining Theorems 3.1 and 4.3 we obtain the Harnack inequality for nonnegative solutions.

Corollary 4.5. Let $u$ be a mesh function satisfying the difference equation

$$
L_{h} u=f
$$

in $\Omega_{h}$, with $u \geq 0$ in $B_{h}$, where $B=B_{R}\left(x_{0}\right) \subset \Omega$. Then for any $\tau<1$, $N h<(1-\tau) R$, we have

$$
\max _{B_{h}^{\tau}} u \leq C\left\{\min _{B_{h}^{\tau}} u+R\left\|\frac{f}{\lambda}\right\|_{n ; B_{h}}\right\},
$$

where $C$ is a positive constant depending on $n, N, \tau, a_{0}$, and $b_{0} R$.

Remark. The proof of Theorem 4.3 , Corollary 4.5 can be extended to permit $c \neq 0$, in which case the constants $C$ also depend on $c_{0} R^{2}$.

Finally, we arrive at an interior Hölder estimate for solutions, which can be deduced from Theorem 4.3 or Corollary 4.5, as in the continuous case [7].

Corollary 4.6. Let $u$ be a mesh function satisfying (4.20) in $\Omega_{h}$. Then for any ball $B=B_{R}\left(x_{0}\right) \subset \Omega$ and $0<\tau<1$, we have

$$
\underset{B_{h}^{\tau}}{\operatorname{osc}} u \leq C \tau^{\alpha}\left\{\left(1+c_{0} R^{2}\right) \max _{B_{h}}|u|+R\|f / \lambda\|_{n ; B_{h}}\right\} \text {, }
$$

where $\alpha$ and $C$ are positive constants depending on $n, N, a_{0}, b_{0} R$.

Remark. By means of a covering argument, we can remove the dependence of $p, \alpha$ on $b_{0} R$ in Theorem 4.3, Corollary 4.6.

\section{BOUNDARY ESTIMATES}

The extensions of the local estimates of the preceding sections to the boundary $\partial \Omega_{h}$ of the set $\Omega_{h}$ proceed analogously to the continuous case [7, §9.9]. In particular, writing for any mesh function $u$ and ball $B \subset \mathbb{R}^{n}$,

$$
\begin{array}{ll}
M=\max _{B_{h}-\Omega_{h}} u, & m=\min _{B_{h}-\Omega_{h}} u, \\
u_{M}^{+}=\max \{u, M\}, & u_{m}^{-}=\min \{u, m\},
\end{array}
$$


we find that Theorems 3.1 and 4.3 continue to hold with $u$ replaced by $u_{M}^{+}$and $u_{m}^{-}$, respectively, and $f$ extended to vanish outside $\Omega_{h}$. From the extended weak Harnack inequality, we can deduce an oscillation estimate in neighborhoods of boundary points: To formulate this, we assume that $\Omega$ satisfies a uniform exterior cone condition, that is, at every point $z \in \partial \Omega$ there exists a finite right circular cone $V_{z}$ with vertex $z$, congruent to a fixed cone $V$ and satisfying $V_{z} \cap \bar{\Omega}=z$. It follows then that there exists a positive number $\theta$ (independent of $h$ ) such that

$$
\frac{1+\left[B_{h}-\Omega_{h}\right]}{\left[B_{h}\right]} \geq \theta
$$

for any ball $B=B_{R}\left(x_{0}\right)$, with center $x_{0} \in \partial \Omega_{h}$. We then have the following estimate.

Theorem 5.1. Let $u$ be a solution of the difference equation (4.20) in $\Omega_{h}$ and $B=B_{R}\left(x_{0}\right)$ be a ball in $\mathbb{R}^{n}$ with center $x_{0} \in \Omega_{h}$. Then for any $0<\tau<1$, we have the estimate

$$
\underset{\Omega_{h} \cap B^{\tau}}{\operatorname{osc}} u \leq C\left\{\tau^{\alpha}\left[\left(1+c_{0} R^{2}\right) \max _{B_{h}}|u|+R\|f / \lambda\|_{n ; \Omega_{h} \cap B_{h}}\right]+\omega(\sqrt{\tau} R)\right\},
$$

where $\alpha$ and $C$ are positive constants depending on $n, N, a_{0}, b_{0} \operatorname{diam} \Omega$, and $\theta$, and $\omega(R)=\operatorname{osc}_{B_{h}-\Omega_{h}} u$.

Note that (5.3) reduces to the interior estimate (4.22) when $B \subset \Omega$.

\section{DiffERENTIAL OPERATORS}

Let $L$ be a linear, second-order differential operator of the form

$$
L u=a^{i j} D_{i j} u+b^{i} D_{i} u+c u,
$$

with real coefficient $a^{i j}\left(=a^{j i}\right), b^{i}, c, i, j=1, \ldots, n$, on the domain $\Omega \subset$ $\mathbb{R}^{n}$. The family of difference operators $\left\{L_{h} \mid 0<h<h_{0}\right\}$, given by (1.2), is called consistent with $L$ if

$$
\lim _{h \rightarrow 0} L_{h} u(x)=L u(x)
$$

for all $u \in C^{2}(\Omega)$ and $x \in \bigcup_{0<h<h_{0}} \Omega_{h}$. Suppose that $L$ is uniformly elliptic in $\Omega$ with bounded coefficients satisfying

$$
\lambda|\xi|^{2} \leq a^{i j} \xi_{i} \xi_{j} \leq \Lambda|\xi|^{2}, \quad|b| \leq \mu, \quad c \leq 0
$$

for all $\xi \in \mathbb{R}^{n}$ and given positive constants $\lambda, \Lambda, \mu$. Then we have the following result of Motzkin and Wasow [11].

Lemma 6.1. There exists a family of positive-type difference operators $\left\{L_{h} \mid 0<\right.$ $\left.h<h_{0}\right\}$ of the form (1.2), consistent with L, satisfying (1.3), (2.1) for $y^{i}=h e_{i}$ 
and $a(x, y)=0$ for $|y|>N h$, where $N, a_{0}, b_{0}, h_{0}$ depend only on $n, \Lambda / \lambda$, and $\mu / \lambda$.

The proof of Lemma 6.1 follows by decomposition of the coefficient matrix $\mathbf{a}=\left[a^{i j}\right]$ into a sum of dyadic matrices,

$$
\mathbf{a}=\sum_{|y|_{\infty}=N} a(y) \tilde{y} \otimes \tilde{y}, \quad \tilde{y}=y /|y|,
$$

with nonnegative coefficients $a(y)=a(\tilde{y})$. This enables us to write the differential operator $L$ in terms of pure second-order derivatives,

$$
L u=\sum_{y} a(y) D_{\tilde{y} \hat{y}} u+b^{i} D_{i} u+c u
$$

yielding the consistent family,

$$
L_{h} u(x)=\sum_{y} a(x, y) \delta_{y}^{2} u(x)+b^{i}(x) \delta_{y^{i}} u(x)+c(x) u(x) \quad\left(y^{i}=h e_{i}\right) .
$$

Let us therefore prove (6.4). In the special case when $\mathbf{a}$ is diagonally dominant, that is,

$$
\sum_{i \neq j}\left|a^{i j}\right| \leq a^{i i}-\lambda_{0}, \quad i=1, \ldots, n
$$

for some constant $\lambda_{0}>0$, we can write

$$
\begin{aligned}
\mathbf{a}= & \sum_{i=1}^{n}\left(a^{i i}-\sum_{i \neq j}\left|a^{i j}\right|\right) e_{i} \otimes e_{i} \\
& +\frac{1}{4} \sum_{i \neq j}\left(\left|a^{i j}\right|+a^{i j}\right)\left(e_{i}+e_{j}\right) \otimes\left(e_{i}+e_{j}\right) \\
& +\frac{1}{4} \sum_{i \neq j}\left(\left|a^{i j}\right|-a^{i j}\right)\left(e_{i}-e_{j}\right) \otimes\left(e_{i}-e_{j}\right),
\end{aligned}
$$

thus obtaining (6.4) with $N=1$ and $a\left(e_{i}\right) \geq \lambda_{0}$. For general a, we first observe that there exists an orthogonal set $y^{1}, \ldots, y^{n}$ with $\left|y^{i}\right|_{\infty}=N$ such that

$$
\left|\tilde{y}^{i}-\varphi^{i}\right|_{\infty} \leq 1 / 2 N
$$

where $\varphi^{1}, \ldots, \varphi^{n}$ is an orthonormal set of eigenvectors of a corresponding to eigenvalues $\lambda_{1}, \ldots, \lambda_{n}$. Consequently,

$$
\begin{aligned}
\mathbf{a} & =\sum_{k=1}^{n} \lambda_{k}\left(\varphi^{k} \otimes \varphi^{k}\right) \\
& =\sum_{k=1}^{n} \lambda_{k}\left(\tilde{y}^{k} \otimes \tilde{y}^{k}\right)+\sum_{k=1}^{n} \lambda_{k}\left(\varphi^{k} \otimes \varphi^{k}-\tilde{y}^{k} \otimes \tilde{y}^{k}\right)
\end{aligned}
$$


will be diagonally dominant with respect to the coordinate system $\tilde{y}^{1}, \ldots, \tilde{y}^{n}$ if

$$
\lambda-n^{2} \Lambda / N \geq \lambda_{0}>0
$$

and the representation (6.4) follows, with

$$
a\left(y^{i}\right) \geq \lambda_{j}-n^{2} \Lambda / N \geq \lambda_{0} .
$$

Note that we can always ensure condition (2.1) for $y^{i}=h e_{i}\left(\right.$ with $\lambda_{i} \geq \lambda / 2$ ), by first replacing a by $\mathbf{a}-\lambda I / 2$.

Using Lemma 6.1, we can pass from Theorems 2.1, 3.1, 4.3, Corollaries 4.5, 4.6, and Theorem 5.1 to their continuous analogues [7, Theorems 9.1, 9.20, 9.22, Corollaries $9.25,9.24,9.28$, respectively]. To accomplish this, we approximate the differential inequalities

$$
L u \geq(\leq) f
$$

for a function $u \in C^{2}(\Omega) \cap C^{0}(\bar{\Omega})$ by difference inequalities,

$$
L_{h} u \geq(\leq) f+\left(L_{h} u-L u\right)
$$

(also approximating $\Omega$ if necessary by a smooth subdomain), and use the fact that the convergence in (6.2) will be uniform in compact subsets of $\Omega$.

The discrete approach thus yields new proofs of these fundamental estimates for differential equations, which are independent of any measure or integration theory. Because of the dependence on $N$, we do not recover the Aleksandrov maximum principle [7, Theorem 9.1] for nonuniformly elliptic operators, but this can be achieved by a refinement of the discrete approach.

\section{BIBLIOGRAPHY}

1. A. D. Aleksandrov, Uniqueness conditions and estimates for the solution of the Dirichlet problem, Vestnik Leningrad. Univ. 18 (1963), no. 3, 5-29; English transl., Amer. Math. Soc. Transl. (2) 68 (1968), 89-119.

2. J. Ya. Bakel'man, Theory of quasilinear elliptic equations, Sibirsk. Mat. Zh. 2 (1961), 179186.

3. J. H. Bramble, B. E. Hubbard, and V. Thomée, Convergence estimates for essentially positive type Dirichlet problems, Math. Comp. 23 (1969), 695-710.

4. J. H. Bramble, R. B. Kellogg, and V. Thomée, On the rate of convergence of some difference schemes for second order elliptic equations, Nordisk. Tidskr. Informationsbehandling (BIT) 8 (1968), 154-173.

5. A. Brandt, Interior estimates for second-order elliptic differential (or finite difference equations) via the maximum principle, Israel J. Math. 7 (1969), 95-121.

6. K P. Bube and J. C. Strikwerda, Interior regularity estimates for elliptic systems of difference equations, SIAM J. Numer. Anal. 20 (1983), 653-670.

7. D. Gilbarg and N. S. Trudinger, Elliptic partial differential equations of second order, 2nd ed., Springer-Verlag, Berlin, Heidelberg, New York, and Tokyo, 1983.

8. N. V. Krylov and M. V. Safonov, ('ertain properties of solutions of parabolic equations with measurable coefficients, Izv. Akad. Nauk. SSSR 40 (1980), 161-175. 
9. H. J. Kuo and N. S. Trudinger, Discrete methods for fully nonlinear elliptic equations (in preparation).

10. A. B. Merkov, Second-order elliptic equations on graphs, Mat. Sb. 127 (1985); English transl., Math. USSR Sb. 55 (1986), 493-509.

11. T. Motzkin and W. Wasow, On the approximation of linear elliptic differential equations by difference equations with positive coefficients, J. Math. Phys. 31 (1953), 253-259.

12. A. V. Pogorelov, The Minkowski multidimensional problem, Wiley, New York, 1978.

13. V. Thomee, Discrete interior Schauder estimates for elliptic difference operators, SIAM J. Numer. Anal. 5 (1968), 626-645.

14. N. S. Trudinger, Local estimates for subsolutions and supersolutions of general second order equations, Invent. Math. 61 (1980), 67-79.

15. __ On regularity and existence of viscosity solutions of nonlinear second order elliptic equations, Partial Differential Equations and the Calculus of Variations: Essays in Honor of Ennio De Giorgi, Birkhäuser, Boston, 1989, pp. 939-957.

Centre for Mathematical Analysis, The Australian National University, G.P.O. Box 4, Canberra, ACT 2601, Australia 